\title{
Research and Design of Wearable Human Motion Capture System
}

\author{
Gang Qin ${ }^{\mathrm{a}}$ and Yi-Xian Chen ${ }^{\mathrm{b}}$ *
}

College of Electrical and Information Engineering, Southwest University for Nationalities, Chengdu,

a616356667@qq.com, bcyxian@hotmail.com

Keywords: Human Motion Capture; Wearable Sensor; Gesture Calculation

\begin{abstract}
This paper analyzes the advantages and disadvantages of the human motion capture, and proposes a wearable human motion capture system based on multi-sensor collaboration. Studying the principle of inertial navigation, through wireless network collaboration form base on multiple sensors (MEMS inertial devices), and using the algorithm based on information fusion technology, accurate human skeleton real-time attitude angle is obtained, completed the task of human motion capture.
\end{abstract}

\section{Introduction}

With the rapid development and popularization of wearable smart devices, more and more wireless transmission technologies, such as WI-FI and Bluetooth, come together on the same platform. At the same time, wearable smart device sets of sensors to make it more powerful, smaller size, lower power consumption. Users use wearable devices will be connected together by wireless sensor technology, access to the Internet of things, to meet the needs of users. This kind of networking and wearable devices brings opportunities and new development direction for the whole market. Nowadays, as the research focus of pattern recognition, human motion recognition technology has been widely used in many fields, such as athlete training, rehabilitation medicine, health monitoring, somatosensory game and movie production. Therefore, it is of great significance and great economic value to design a wearable human motion capture system which is easy to use, free to monitor, and does not affect the daily activities of human beings.

\section{Human Motion Capture}

Human motion capture technology attracts more and more scientific research institutions and related companies because of its extensive application fields and huge market development prospects. At present, human motion capture technology is divided into non-wearable motion capture and wearable motion capture.

Non-wearable motion capture system can achieve high accuracy in the measurement range, the system through the external power supply, can continuously capture human motion information. But there are some shortcomings, such as limited range of measurement, that is, only the object which be measured is in the scope of measuring range of the capture device. Different methods of non-wearable technology have their own shortcomings, such as the key points of the motion capture system based on optical image recognition technology were obscured, electromagnetic interference problem of positioning and tracking motion capture system based on electromagnetic etc.

The advantages of wearable motion capture system are easy to wear, miniaturization, low power consumption and low cost. It collects less data, so the information processing time is less, and it is less affected by environmental factors in the process of using. However, in earlier studies, people usually used single sensor signals (such as accelerometers, gyroscopes, or magneto resistive sensors) to capture human movements. Among them, acceleration signals are most frequently used. And these studies are only for specific postures of the human body (for example, the fall detection of the elderly), and the function is too single. 
In order to solve these problems, researchers have proposed a variety of motion gesture recognition algorithm which is more comprehensive and more perfect. Wee-Soon designed a measurement algorithm of human posture, which through the three wearable inertial measurement units were deployed in the waist and lower limb, to achieve real-time discrimination on the human body lying down, sitting and standing. LIW presented a fusion algorithm of DS evidence theory based on the data in the static environment using the body area network system to collect multiple acceleration signal. HE Z use acceleration sensor which is placed in the crotch to obtain human motion information, and use the classification method of support vector machine to realize the static walking, running and jumping.

\section{Design Scheme}

Whole Scheme. A wearable human motion capture system is designed with four degrees of freedom simplified human model. The system is based on the principle of inertial navigation, composed by multiple MEMS sensors, and use information fusion algorithm. It complete the task of human motion capture base on the accurate human skeleton real-time attitude angle.

The system consists of 9 MEMS sensor measurement units, each measuring unit is equipped with an mpu9250 sensor, and the Bluetooth chip Nrf51822 is used as MCU. After collecting sensor data, MCU fuse the data base on gradient descent algorithm, while using the Kalman filtering algorithm to filter the data. It obtain a more accurate and more easily with the attitude data, to achieve a stable output data of high precision attitude angle.

Communication of the system based on Bluetooth 4.1 protocol, it upload the attitude data to the computer, the computer analyses human body model and draws a three-dimensional model by the PC program, it real-time controls the human body model, to achieve real-time human motion reconstruction, through key frame animation technology, and using the attitude data real-time receiving. System architecture diagram as shown in Fig. 1

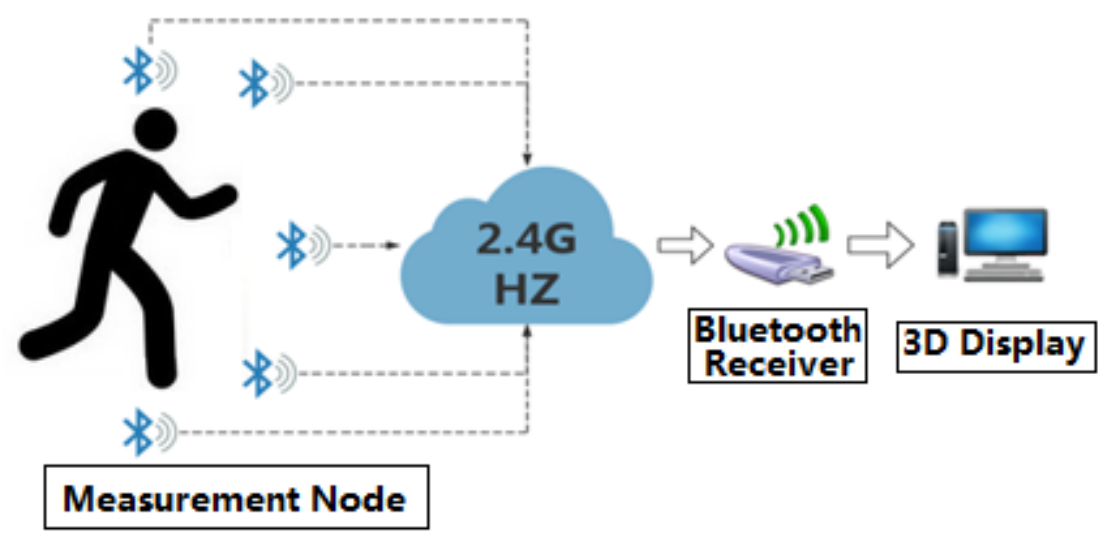

Figure 1. System architecture diagram

Hardware Structure. Processor module. Because the wearable system must be compact and lightweight, the physical size and quality of the whole system must be considered in the process of designing the system. In addition, in order not to affect the human movement, power supply must be battery powered, and the whole system's endurance is limited to the nodes with the lowest power. It also means that communications must be considered in a wireless manner, and nodes and the entire system must be designed for low power consumption. Therefore, an integrated nRF51x series wireless transceiver (Soc) which is ultra-low power chip is used as MCU to meet the design requirements.

Sensor Module. The MPU9250 nine axis sensors is selected as human motion capture sensors. The chip can also open the low power function alone, allowing the host to enter the sleep mode, thus greatly reducing the energy consumption.

Software structure. 9 slaves and 1 master networking by the Bluetooth communication protocol in NRF51822, analyses were conducted on the data those are collected from nine inertial 
measurement unit, and the results are transmitted to the Bluetooth transceiver by the communication module and UART serial port. After verification of encoding data frame, the host computer process the data those are transmitted by the serial communication module. The system flowchart as shown in Fig. 2.

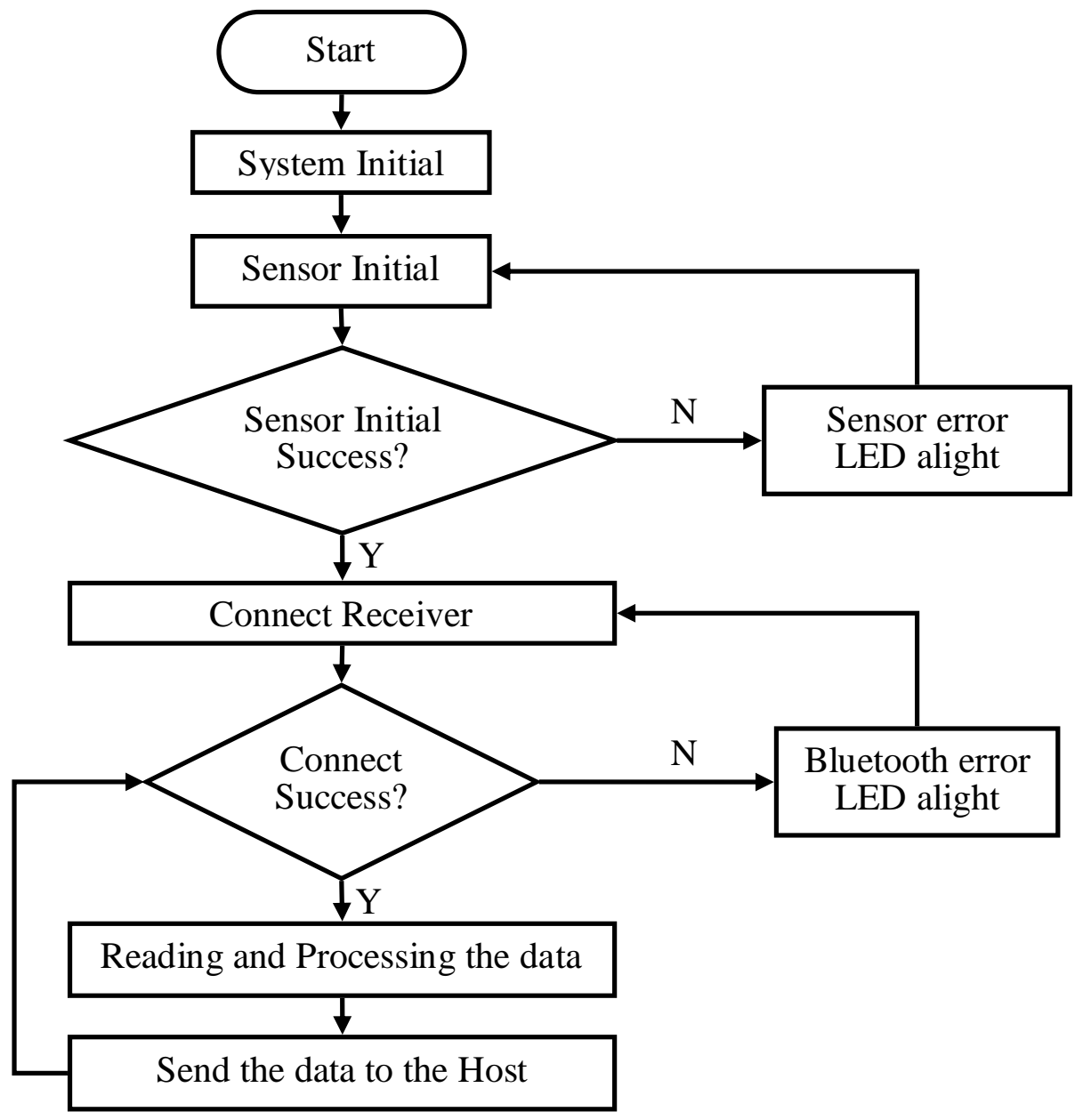

Figure 2. System Flowchart

The MPU9250 collect the state data of 3 axis acceleration, 3 axis and 3 axis gyro magnetic meter data, and then use the gradient descent algorithm to fuse those data of three types. At the same time use the Kalman filtering algorithm to filter the data to improve accuracy of attitude data, attitude calculation process is shown in Fig. 3.

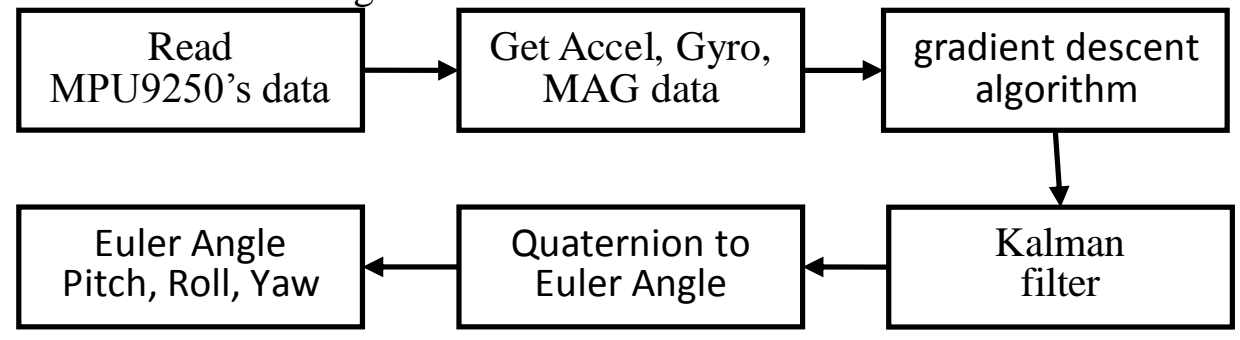

Figure 3. Attitude Calculation Flowchart

\section{Conclusions}

Study of the design process, the wearable human motion capture system has the characteristics of small size and portability. A single module is designed with four layers, making each module the same size as a coin, as shown in figure 6. Moreover, it uses the gradient descent algorithm to merge the original data, and uses the Calman filtering algorithm to filter the data, improve data accuracy and real-time, achieve the real-time control of the human body model requirements. Finally, on the 
basis of the compact design of a single module, in order to wear comfortable, it put the equipment with the wrist, knee, waistband together, as shown in Fig. 4 and Fig. 5.

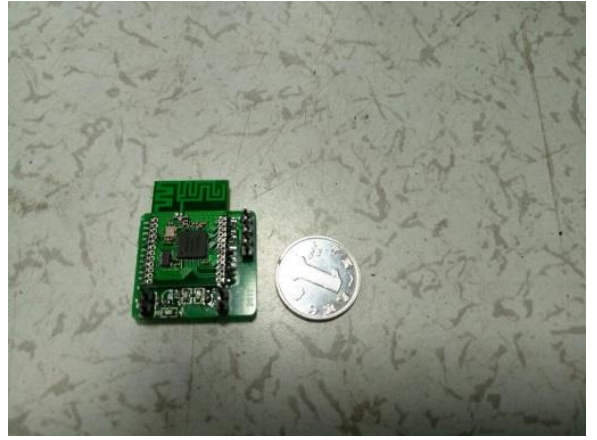

Figure 4. A Single Measurement Module

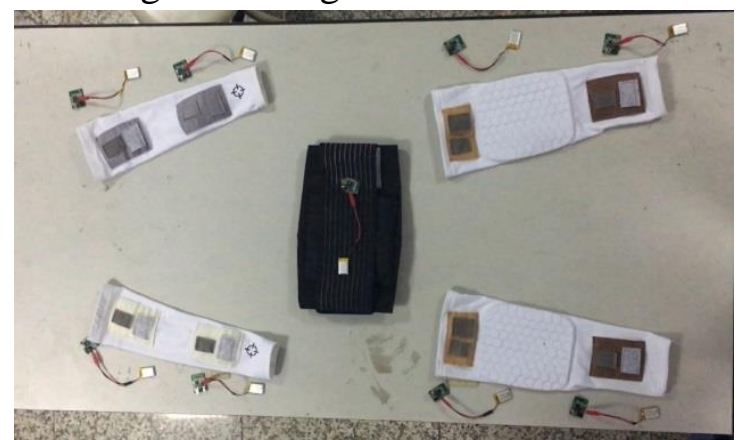

Figure 5. System Appearance

\section{Acknowledgements}

This work was financially supported by the innovative training program for college students, Southwest Minzu University (201710656009)

\section{References}

[1] DONG Yuhua, SUN Yanhui, XU Guokai. SONG Peng, Research on Gesture Recognition Based on ZigBee and Acceleration Sensor[J], Chinese Journal of Sensors and Actuators, 2013, Vol.26(7):961-965.

[2] Guo Zhihu, FeiJie, Qian Feng. Research of Human Motion Capture System Based on the Whole Attitude of the Micro-Inertial Measurement[J], Microcomputer Applications, 2013, Vol.29(2):29-32.

[3] RYAN A, JOSEPH A P. A wireless, compact, multi-user sensor system for interactive dance[C], Proceedings of the 2006 international conference on new interfaces for musical expression, Paris, France, 2006:134-139.

[4] Ferscha A, Resmrita S. Gestural Interaction in the Pervasive Computing Landscape[J]. E \& I Elektrotechnik Und Informationstechnik, 2007,124(1):17-25.

[5] Lee Y,Kim J, Son M, et al. Implementation of accelerometer sensor module and fall detection monitoring system base on wireless sensor network[C], Engineering in Medicine and Biology Society, 2007. EMBS 2007. 29th Annual International Conference of the IEEE. IEEE,2007:2315-1318. 